\title{
Performance Analysis of Intensity Modulation Techniques in Atmospheric Turbulent Channels
}

\author{
Sara S. Abd El-Hak \\ A National Center for Radiation \\ Research and Technology \\ (NCRRT), Egyptian Atomic Energy \\ Authority (EAEA), Cairo 11787, \\ Egypt
}

\author{
Abdulaziz E. Elfiqi \\ Dept. of Communications \\ Faculty of Electronic Engineeing, \\ Menofia University \\ Menouf, Egypt
}

\author{
F. E. Abd El-Samie \\ Dept. of Communications \\ Faculty of Electronic Engineeing. \\ Menofia University \\ Menouf, Egypt
}

\author{
Ahmed E. Morra \\ Dept. of Communications \\ Faculty of Electronic Engineeing, \\ Menofia University \\ Menouf, Egypt
}

\begin{abstract}
In this paper, we introduce a study for the performance of intensity modulation (IM) techniques over gamma-gamma (GG) distributed free-space optical (FSO) channels. We derived closed form expressions for the average bit-error rate (BER) of thermal noiselimited FSO systems adopting various IM techniques. The effects of the atmospheric turbulence and the propagation wavelength are studied. A comparison among different IM techniques in term of transmitted peak power to achieve certain BER is presented. Results show that OOK has the best BER compared with other IM techniques. In addition, according to peak power, PPM and MPPM are more power efficient than DPPM and DMPPM, respectively.
\end{abstract}

\section{Introduction}

Recently, free-space optical (FSO) communications has received great attention due to its advantages over radio frequency (RF) communications. These advantages include wide modulation bandwidth, inherent security, immunity to electromagnetic interference and license free [1-3]. These great advantages promote the FSO transmission to be used along with the RF one in different communication networks, i.e, fifth generation(5G) mobile networks [4-6]. However, FSO transmission is very sensitive to weather conditions, such as fog attenuation and scintillation (i.e., atmospheric turbulence) [7]. In order to overcome the scintillation effect, power efficient modulation techniques should be used [8]

Intensity modulation (IM) techniques are mostly used with optical wireless communications due to their simplicity and power efficiency [2]. IM techniques include on-off keying (OOK) [3], pulse position modulation (PPM) [9],[10], multipulse-pulse position modulation (MPPM), differential PPM (DPPM) [11] and differential MPPM (DMPPM) [12]. OOK is the dominant scheme used in commercial FSO communication systems. In OOK signaling, a transmitted optical pulse represents " 1 " and the optical absence represents " 0 ". It needs a threshold level to detect the transmitted bits at the receiver side [13].
One of the most power efficient techniques which can be detected using soft detection is PPM [14]. In this scheme, the transmission time is divided into equal consecutive frames which represents PPM symbols. Each PPM symbol is divided again into $\mathrm{Q}$ equal time slots where only one optical pulse is transmitted during one of the Q time slots in each signal frame. The information is represented by the position of the pulse in each signal frame as in Fig. 1(a). Therefore, $\log _{2}(Q)$ bits are transmitted during every PPM symbol [8]. In order to improve the spectral efficiency of PPM, MPPM was proposed at the expense of increasing the encoding complexity and decreasing the power efficiency [14]. In MPPM scheme, $\mathrm{K}$ optical pulses are transmitted in multiple slots in a frame of Q time slots length has in Fig. 1(b). Thus, $\left(\begin{array}{l}Q \\ K\end{array}\right)$ symbols can be formed and transmitted, in other words $\log _{2}\left(\begin{array}{l}Q \\ K\end{array}\right)$ bits of information can be transmitted per MPPM symbol [ 8].

DPPM and DMPPM are modifications of PPM and MPPM, respectively, where the symbol length is allowed to vary as in Fig. 1(d) and (c), by removing the "0"s after the last "1" to get higher spectral efficiencies compared with the PPM and MPPM.

BER performance of OOK was discussed with space diversity reception technique (SDRT) over gamma-gamma atmospheric turbulence transmission but without a clear final expression of average BER in [15]. Adaptive threshold OOK was studied over log-normal distribution which describes fading under weak atmospheric turbulence in [16]. The BER as a function of average power were studied using negative exponential distribution which models strong turbulence in [17]. The performance analysis of PPM and MPPM techniques under gamma-gamma distribution of FSO thermal noise limited and shot noise system were investigated in [1].

In this paper, we compare the BER performance of different IM techniques under different operating and weather conditions to make a guideline for using the IM techniques under FSO channels. In addition, some of the IM 
techniques (i.e. DPPM and DMPPM) do not have closed form bit error rate (BER) expressions under gamma-gamma (GG) turbulent FSO channels, thus, we derive closed form expressions of BER for thermal noise-limited FSO systems. The effect of changing the operating laser source wavelength on the performance of these systems are investigated under different atmospheric conditions. Moreover, comparison among various techniques is in term of peak power to achieve certain BER performance.

The rest of the paper is organized as follows. In Sections 2 and 3, BER expression is explored for nonturbulent channels and PAPR is discussed for different IM techniques, respectively. In Section 4, expressions for the union bound of the average BER of thermal noise-limited systems over gamma-gamma channels is derived, respectively. The performance of IM techniques is numerically investigated and compared in term of peak transmitted power under different atmospheric conditions in Section 5. Finally, the conclusions are drawn in Section 6.

\section{BER Performance under Non-Turbulent Channels}

To identify the differences among the different intensity modulation schemes, we study the performance of these modulation techniques under non-turbulent channels. The bit error rate (BER) can be expressed as [18]

$$
\text { BER } \leq \frac{M}{4} \operatorname{erfc}\left(\frac{d_{\text {min }}}{2 \sqrt{N_{0}}}\right)
$$

Where the minimum Euclidean distance between two symbols is $d_{\min }$, the power spectral density of the noise is $\mathrm{N}_{0} / 2$ and the cardinality of the constellation is M. Ageneral BER expression for different IM schemes can be written as

$$
\mathrm{BER}_{\mathrm{IM}} \leq \mathrm{B} \operatorname{erfc}\left(\mathrm{C} \sqrt{\gamma_{\mathrm{k}}}\right)
$$

WhereB and $\mathrm{C}$ depend on the type of the modulation scheme and are given in Table 1 and the $\boldsymbol{\gamma}_{\mathbf{k}}$ average signalto-noise ratio (SNR) which is expressed as [1],

$$
\gamma_{k}=\frac{\mathbf{R}^{2} \mathbf{P}_{\mathrm{av}}^{2}}{\sigma_{\mathrm{n}}^{2}}
$$

where $\boldsymbol{P}_{\mathbf{a v}}$ is the average received optical power, $\boldsymbol{\sigma}_{\mathbf{n}}^{\mathbf{2}}$ is the additive white Gaussian receiver noise variance andRis the photodiode responsivity.

\section{Peak-to-Average Power Ratio Analysis}

Due to the limited dynamic range of real communication systems, peak-to-average power ratio (PAPR) is an important metric to compare different modulation techniques [22]. High PAPR causes signal distortion and leads to performance degradation [22].

In Table 2, different expressions of PAPR required for each IM scheme are listed. It can be noticed from Table 2 that PPM based schemes, increasing number of time slots per frame, Q, leads to decrease the average power and as a results the PAPR increases. Furthermore, increasing the number of optical pulses per frame, $\mathrm{K}$, decreases the PAPR because of decreasing the peak power as in MPPM. For OOK scheme, PAPR depends on the cardinality level, $\mathrm{M}$, which is constant for OOK scheme (i.e:, $M=2$ ). Both DMPPM and DPPM provide lower PAPR compared to MPPM and PPM, respectively, for the same frame length, $Q$ because of the variable symbol length.

\section{BER Under Gamma-Gamma Turbulent FSO Channels}

In this section, we derive union bound Bit-error rate (BER) expressions of thermal noise-limited intensity modulated (IM) systems over gamma-gamma turbulence FSO channel. Optical transmission suffers from two fundamental noise sources that affect FSO receivers: shot noise (which originates from input signals or background radiation) and thermal noise (which arises from photo detector load resistor and amplifier noises) [23]. Shot noise dominates over thermal noise only if received power is high. This could happen if transmitter and receiver are close to each other in some optical networks. Otherwise, shot noise could be neglected. Mostly, the received power is low at most of the receivers where thermal noise dominates the shot noise. In addition, a PIN-based receiver is more common to be used in IM systems and it is usually thermal-noise limited [2]. In this section, we drive union bound symbol error rate expression of thermal-noise limited IM system over gamma-gamma turbulence FSO channel. Gamma-gamma model has gained a wide acceptance because it is suitable for strong and weak atmospheric turbulences [7]. This model can be adjusted by two parameters ( $\alpha$ and $\beta$ ) which are the effective numbers of large and smallscale eddies of the scattering environment and those parameters basically depend on the Rytovvariance $\sigma_{R}^{2}$.

\subsection{GG Turbulence FSO Channel Model}

GG distribution has gained a wide validation for strong and weak atmospheric turbulences [24]. The probability density function (PDF) of GG distribution is given as [27]

$$
f(h)=\frac{2(\alpha \beta)^{\frac{\alpha+\beta}{2}}}{\Gamma(\alpha) \Gamma(\beta)} h^{\frac{\alpha+\beta}{2}-1} J_{\alpha-\beta}(2 \sqrt{\alpha \beta h})
$$

where $\mathrm{h}$ is the FSO channel state gain i.e: $\mathrm{h} \geq 0, \Gamma(\bullet)$ donates Gamma function, $\mathrm{Jx}(\bullet)$ is the second kind 
modified Bessel function of order $\mathrm{x}$. The scintillation parameters, $\alpha$ and $\beta$, are expressed as [25]

$$
\begin{aligned}
\alpha & =\left(\exp \left[\frac{0.49 \sigma_{\mathrm{R}}^{2}}{\left(1+1.11 \sigma_{\mathrm{R}}^{12 / 5}\right)^{7 / 6}}\right]-1\right)^{-1}, \\
\beta & =\left(\exp \left[\frac{0.51 \sigma_{\mathrm{R}}^{2}}{\left(1+0.69 \sigma_{\mathrm{R}}^{12 / 5}\right)^{5 / 6}}\right]-1\right)^{-1},
\end{aligned}
$$

respectively, where $\sigma_{\mathrm{R}}^{2}=1.23 \mathrm{C}_{\mathrm{n}}^{2}\left(\frac{2 \pi}{\lambda}\right)^{7 / 6} \mathrm{~L}^{11 / 6}$ with $\mathrm{C}_{\mathrm{n}}^{2}$ is the refractive-index structure parameter, $\mathrm{L}$ is FSO propagation distance and $\lambda$ is the operating optical wavelength. Based on link equation of FSO channel in [26], the average received power as a function of FSO system parameters can be expressed as

$$
\mathrm{P}_{\mathrm{r}}(\mathrm{h})=\zeta_{\mathrm{t}} \mathrm{G}_{\mathrm{t}} \zeta_{\mathrm{r}} \mathrm{G}_{\mathrm{r}}\left(\frac{\lambda}{4 \pi \mathrm{L}}\right)^{2} h \mathrm{P}_{\mathrm{t}}
$$

where $P_{t}$ is the average transmitted power, $\zeta_{t}$ and $\zeta_{r}$ are the efficiencies of transmitter and receiver, respectively, $G_{t}$ and $G_{r}$ are the telescope gains of transmitter and receiver, respectively. For simple analysis, we assume identical transceivers systems, i.e.: $\zeta_{\mathrm{t}}=\zeta_{\mathrm{r}}=\zeta$ and $\mathrm{G}_{\mathrm{t}}=\mathrm{G}_{\mathrm{r}}=\mathrm{G} \approx(\pi \mathrm{D} / \lambda)^{2}$, where $\mathrm{D}$ is the telescopic diameter [28]. Thus, the average received power can be simplified to

$$
\mathrm{P}_{\mathrm{r}}(\mathrm{h})=\left(\frac{\zeta \mathrm{A}}{\lambda \mathrm{L}}\right)^{2} \mathrm{hP}(8)
$$

where $\mathrm{A}=\pi D^{2} / 4$ is the aperture area. Therefore, the average signal-to-noise ration can be expressed as a function of the FSO channel gain as

$$
\gamma_{k}(h)=R^{2}\left(\frac{\zeta A}{\lambda L}\right)^{4} h^{2} \frac{P_{t}^{2}}{\sigma_{n}^{2}}(9)
$$

\subsection{Union-Bound BER of IM Techniques under FSO Turbulence Channels}

The average BER for different IM techniques can be calculated following a similar approach as [1]:

$$
\mathrm{BER}_{\mathrm{IM}}^{\mathrm{av}}=\int_{0}^{\infty} B E R_{\mathrm{IM}} \mathrm{f}(\mathrm{h}) \mathrm{dh}
$$

Substituting (2) and (4) into (10), we get

$$
\mathrm{BER}_{\mathrm{IM}}^{\mathrm{av}} \leq \frac{2 \mathrm{~B}(\alpha \beta)^{\frac{\alpha+\beta}{2}}}{\Gamma(\alpha) \Gamma(\beta)} \int_{0}^{\infty} \times \operatorname{erfc}\left(\mathrm{C} \sqrt{\gamma_{\mathrm{k}}(\mathrm{h})}\right) d h
$$

By expressing $\operatorname{erfc}(\cdot)$ using Meijer-G function [27] and $J_{\alpha-\beta}(\bullet)$ using [27], we get

$$
\begin{gathered}
\operatorname{BER}_{\mathrm{IM}}^{\mathrm{av}} \leq \frac{\mathrm{B}(\alpha \beta)^{\frac{\alpha+\beta}{2}}}{\sqrt{\pi} \Gamma(\alpha) \Gamma(\beta)} \\
\times \int_{0}^{\infty \mathrm{h}^{\frac{\alpha+\beta}{2}-1} \mathrm{G}_{0,2}^{2,0}\left(\alpha \beta \mathrm{h} \mid \frac{\alpha-\beta}{2}, \frac{-\alpha+\beta}{2}\right)} \mathrm{dh} \\
\mathrm{G}_{1,2}^{2,0}\left(\left.\mathrm{C}^{2} \gamma_{\mathrm{k}}(\mathrm{h})\right|_{0,0.5} ^{1,-}\right)
\end{gathered}
$$

By substituting of $\gamma_{k}(h)$ from (9) into (12) and using Meijer-G function integral expressions in [27], a closedform expressions for union bound of average BER over FSO channels of intensity modulation schemes can be expressed in (13), where C and B can be obtained from Table 1.

$$
\begin{aligned}
& \mathrm{BER}_{\mathrm{IM}}^{\mathrm{av}} \leq \frac{\mathrm{B} 2^{\alpha+\beta-2}}{\pi^{3 / 2} \quad \Gamma(\alpha) \Gamma(\beta)} \mathrm{G}_{5,2}^{2,4} \\
& \times\left(\left(4 \mathrm{C} \frac{\mathrm{R}}{\alpha \beta}\left(\frac{\zeta \mathrm{A}}{\lambda \mathrm{L}}\right)^{2} \frac{\mathrm{P}_{\mathrm{t}}}{\sigma_{\mathrm{n}}}\right)^{2} \mid \frac{1-\beta}{2}, \frac{2-\beta}{2}, \frac{1-\alpha}{2}, \frac{2-\alpha}{2}, 1\right)
\end{aligned}
$$

\section{Numerical Results}

In this section, comparison between performance of thermal noise-limited FSO systems adopting different intensity modulation techniques is presented in terms of transmitted peak power and average transmitted power per bit using Gamma-Gamma distribution for weak, moderate and strong turbulences $\left(\mathrm{C}_{\mathrm{n}}^{2}=5.2 \times 10^{-16} \mathrm{~m}^{-2 / 3}, \mathrm{C}_{\mathrm{n}}^{2}=2.1 \times 10^{-15} \mathrm{~m}^{-2 / 3}\right.$ and $\mathrm{C}_{\mathrm{n}}^{2}=1.3 \times 10^{-14} \mathrm{~m}^{-2 / 3}$, respectively) and two operating wavelengths $(850 \mathrm{~nm}$ and $1550 \mathrm{~nm})$ for the same bandwidth and comparable bit rates (M-ary PPM $(\mathrm{M}=7)$, DPPM $(\mathrm{M}=$ 23), $\operatorname{MPPM}(20,2), \operatorname{DMPPM}(54,2))$.

\subsection{Peak power-limited scenario}

Increasing the transmitted peak power increases the signal to noise ratio and the performance of the communication system improves. However, there are some constraints on the peak power related to the dynamic range of the source to limit the non-linear distortion caused form high peak power [28]. The modulation technique that can achieve high performance at lower peak power is desirable.

Figure2showstheperformanceofIMmodulationschemes in term of transmitted peak power $(\mathrm{dBm})$. We plot the average BER versus transmitted peak power $(\mathrm{dBm})$ at wavelength $(\lambda=1550 \mathrm{~nm})$ and at different atmospheric turbulence levels with noise variance $\sigma_{n}^{2}=$ $25 \times 10^{-14} A^{2}$.As shown in Table 3 , 


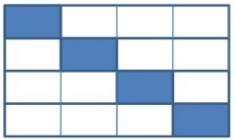

a) $\operatorname{PPM}(\mathrm{Q}=4)$

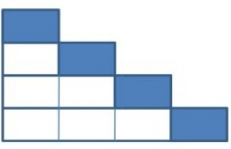

d) $\operatorname{DPPM}(\mathrm{Q}=4)$

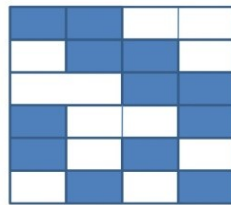

b) $\operatorname{MPPM}(\mathrm{Q}=4, \mathrm{~K}=2)$

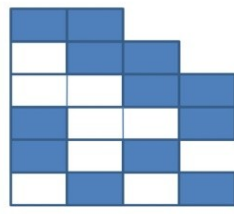

c) $\operatorname{DMPPM}(\mathrm{Q}=4, \mathrm{~K}=2)$

Figure1: Representation of 2 bits symbols for different modulation techniques

Table 1: BER Parameters for Different IM Techniques

\begin{tabular}{cccc}
\hline Scheme & B & C & References \\
\hline OOK & $\frac{1}{2}$ & $\frac{1}{\sqrt{2}}$ & {$[3],[13]$,} \\
PPM & $\frac{Q}{4}$ & $\frac{Q}{2}$ & {$[19],[20]$} \\
DPPM & $\frac{(Q+1)}{8}$ & $\frac{(Q+1)}{4}$ & {$[20],[19]$,} \\
MPPM & $\frac{\left(\begin{array}{l}Q \\
K\end{array}\right)}{4}$ & $\frac{Q}{2 K}$ & {$[21],[12],[3]$} \\
DMPPM & $\left.\frac{\left(\frac{Q+K}{2}\right.}{K}\right)$ & $\frac{Q+K}{4 K}$ & {$[12]$} \\
& $\frac{}{4}$ & & \\
\hline
\end{tabular}

Table 2: Peak-to-average power ratio (PAPR) of various intensity modulation schemes.

\begin{tabular}{cc}
\hline Scheme & PAPR \\
\hline OOK & 2 \\
PPM & $Q$ \\
DPPM & $\frac{(Q+1)}{2}$ \\
MPPM & $\frac{Q}{K}$ \\
DMPPM & $\frac{Q+K}{2 K}$ \\
\hline
\end{tabular}

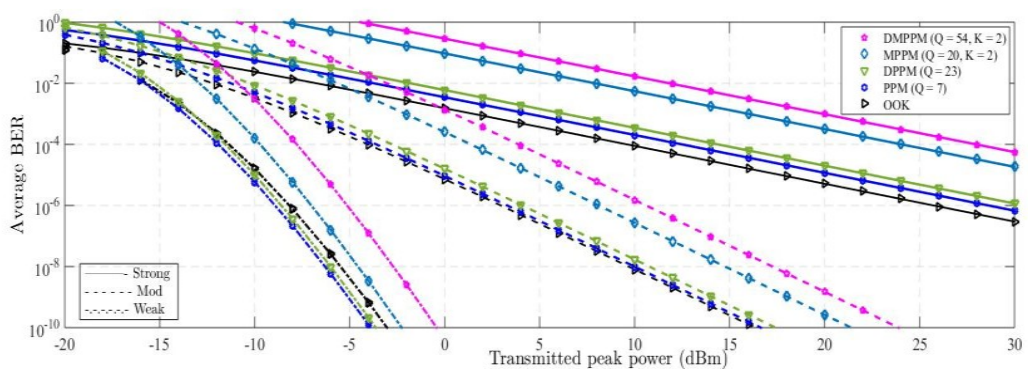

Figure 2: Average BER versus transmitted peak power $(\mathrm{dBm})$ for IM modulation schemes at different atmospheric turbulence levels at wavelength $=1550 \mathrm{~nm}$ and $\sigma_{n}^{2}=25 \times 10^{-14} A^{2}$ 
Table 3: Peak powerof IM schemes with the same data rate at a BER of $10^{-6}$.

\begin{tabular}{cccc}
\hline Scheme & $\begin{array}{c}\mathrm{P}_{\text {peak } 1}(\mathrm{dBm}) \\
\text { Weak Turbulence }\end{array}$ & $\begin{array}{c}\mathrm{P}_{\text {peak 2 }}(\mathrm{dBm}) \\
\text { Moderate Turbulence }\end{array}$ & $\begin{array}{c}\mathrm{P}_{\text {peak } 3}(\mathrm{dBm}) \\
\text { Strong } \\
\text { Turbulence }\end{array}$ \\
\hline OOK & -8.25 & 3 & 25.6 \\
PPM & -8.9 & 3.25 & 28.5 \\
DPPM & -8.6 & 4 & 30.5 \\
MPPM & -7 & 8 & 40 \\
DMPPM & -5.1 & 4.5 & 44 \\
\hline
\end{tabular}

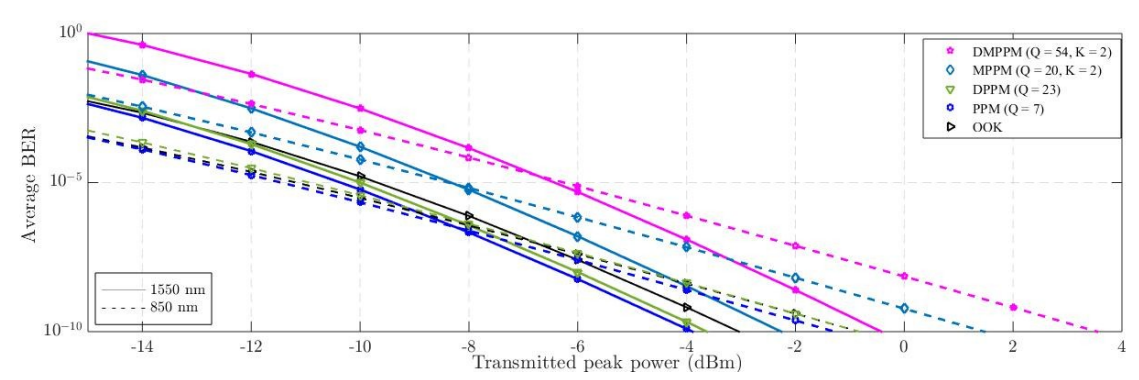

Figure 3: Comparison between average BER of IM modulation schemes at weak atmospheric turbulence at wavelength $=1550$ and $850 \mathrm{~nm}$ and $\sigma_{n}^{2}=25 \times 10^{-14} A^{2}$

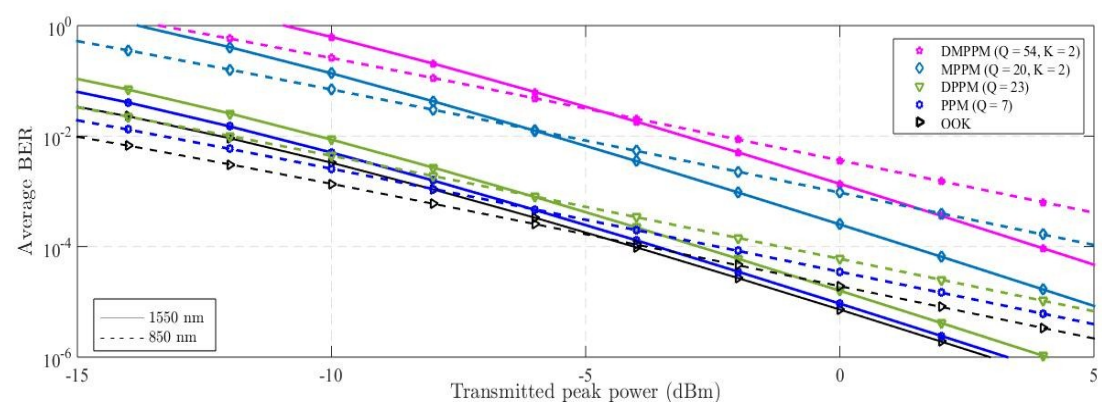

Figure 4: Comparison between average BER of IM modulation schemes at moderate atmospheric turbulence at wavelength $=$ 1550 and $850 \mathrm{~nm}$ and $\sigma_{n}^{2}=25 \times 10^{-14} A^{2}$

Table 4: Average BER of IM schemes at weak and moderate atmospheric turbulences at low and high peak power.

\begin{tabular}{|c|c|c|c|c|c|c|c|c|}
\hline \multirow{3}{*}{ Scheme } & \multicolumn{4}{|c|}{$\begin{array}{c}\text { Weak turbulence } \\
C_{n}^{2}=5.2 \times 10^{-16} \mathrm{~m}^{-2 / 3}\end{array}$} & \multicolumn{4}{|c|}{$\begin{array}{l}\text { Moderate turbulence } \\
C_{n}^{2}=2.1 \times 10^{-15} \mathrm{~m}^{-2 / 3}\end{array}$} \\
\hline & \multicolumn{2}{|c|}{$\begin{array}{l}P_{11}=-14 \\
(\mathrm{dBm})\end{array}$} & \multicolumn{2}{|c|}{$(\mathrm{dBm}){ }^{P_{12}=-4}$} & \multicolumn{2}{|c|}{$\begin{array}{c}P_{21}=-10 \\
(\mathrm{dBm})\end{array}$} & \multicolumn{2}{|c|}{$P_{22}=0$} \\
\hline & $\begin{array}{l}\text { BER at } \\
850 \mathrm{~nm}\end{array}$ & $\begin{array}{l}\text { BER at } \\
1550 \mathrm{~nm}\end{array}$ & $\begin{array}{l}\text { BER at } \\
850 \mathrm{~nm}\end{array}$ & $\begin{array}{l}\mathrm{BER} \text { at } \\
1550 \mathrm{~nm}\end{array}$ & $\begin{array}{l}\mathrm{BER} \text { at } \\
850 \mathrm{~nm}\end{array}$ & $\begin{array}{c}\text { BER at } \\
1550 \mathrm{~nm})\end{array}$ & $\begin{array}{l}\text { BER at } \\
850 \mathrm{~nm}\end{array}$ & $\begin{array}{l}\text { BER at } \\
1550 \mathrm{~nm}\end{array}$ \\
\hline OOK & $1 \times 10^{-4}$ & $3 \times 10^{-3}$ & $1 \times 10^{-4}$ & $1 \times 10^{-4}$ & $1 \times 10^{-3}$ & $4 \times 10^{-3}$ & $2 \times 10^{-5}$ & $7 \times 10^{-6}$ \\
\hline PPM & $1 \times 10^{-4}$ & $1 \times 10^{-3}$ & $1 \times 10^{-4}$ & $1 \times 10^{-4}$ & $2 \times 10^{-3}$ & $5 \times 10^{-3}$ & $3 \times 10^{-5}$ & $9 \times 10^{-5}$ \\
\hline D-PPM & $2 \times 10^{-4}$ & $3 \times 10^{-3}$ & $1 \times 10^{-4}$ & $1 \times 10^{-4}$ & $4 \times 10^{-3}$ & $8 \times 10^{-3}$ & $6 \times 10^{-5}$ & $1 \times 10^{-5}$ \\
\hline MPPM & $3 \times 10^{-3}$ & 0.04 & $1 \times 10^{-4}$ & $1 \times 10^{-4}$ & 0.06 & 0.13 & $9 \times 10^{-4}$ & $2 \times 10^{-4}$ \\
\hline D-MPPM & 0.028 & 0.415 & $1 \times 10^{-4}$ & $1 \times 10^{-4}$ & 0.2 & 0.6 & $3 \times 10^{-3}$ & $1 \times 10^{-3}$ \\
\hline
\end{tabular}




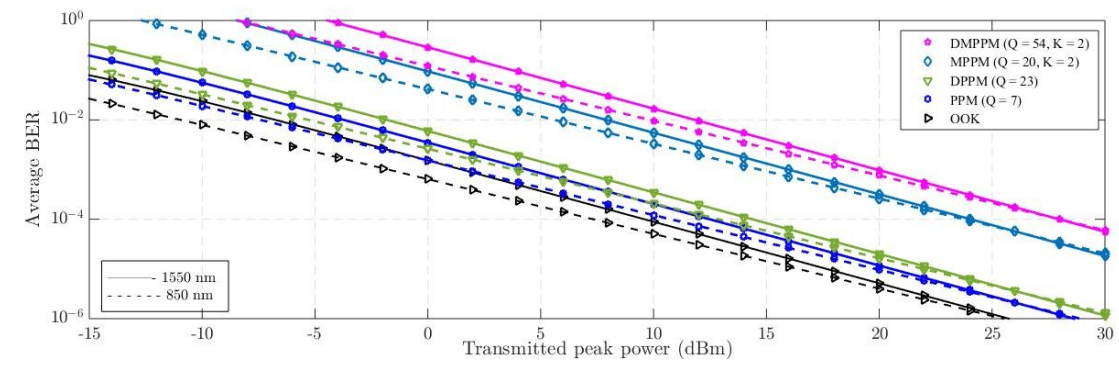

Figure 5: Comparison between average BER of IM modulation schemes at strong atmospheric turbulence $\mathrm{C}_{\mathrm{n}}^{2}=$ $1.3 \times 10^{-14} \mathrm{~m}^{-2 / 3}$ at wavelength $=1550$ and $850 \mathrm{~nm}$ and

$$
\sigma_{n}^{2}=25 \times 10^{-14} A^{2}
$$

To achieve average BER $\left(10^{-6}\right)$, at weak turbulence, DMPPM requires the highest peak power per pulse compared to other techniques, approximately $2 \mathrm{dBm}$ higher than MPPM for the same transmitted data rate because the varying length of the symbol increases the error probability, thus, DMPPM requires higher peak power to achieve the same performance compared to other IM techniques. Although, MPPM, DPPM provide higher spectral efficiency more than PPM but this comes at the cost of higher peak power. When atmospheric turbulence level increase, the behavior of IM schemes doesn't change dramatically. But it needs higher peak power to achieve the same average BER performance to overcome the effect of this turbulence level increases. Moreover, PPM still provide the best performance compared to others for all values of $C_{n}^{2}$.

Figures 3 and 4represent the effect of changing wave length on the performance of the system at weak, moderate atmospheric turbulences.

As shown in table4, the main observation is, at the same value of $C_{n}^{2}$ when the power is low, the effect of noise on system performance dominates the turbulence effect. As a result, the performance of all schemes operated at wavelength $850 \mathrm{~nm}$ is better than their performance at 1550 $\mathrm{nm}$ because the telescopic gain is inversely proportional with the square of the operational wavelength. However, at a certain point, as the power increases the performance at $1550 \mathrm{~nm}$ becomes better than the performance at $850 \mathrm{~nm}$. Because at high power, turbulence dominates and the transmitted signals at higher wavelength suffer less turbulence than signals transmitted at short wavelength because of the inverse relationship between $\sigma_{R}^{2}$ and $\lambda^{7 / 6}$. Figure 5represent the effect of changing wave length on the performance of the system at strong turbulence. It shows that changing the wavelength doesn't provide a significant effect on performance of the system at strong turbulence.

\section{Conclusion}

Closed form expressions for average BER of thermal noiselimited FSO systems adopting different intensity modulation (IM) techniques have been driven over gamma-gamma (GG) distribution. Comparison among different IM techniques under different atmospheric turbulence levels has been investigated in term of transmitted peak power. Our results show that, although DPPM and DMPPM provide lower PAPR, their variable length frames increase their probability of error.
In addition, according to peak power, PPM and MPPM are more power efficient than DPPM and DMPPM, respectively. For example, at weak turbulence, PPM saves peak power around $0.3(\mathrm{dBm})$ and MPPM saves around 1.9 $(\mathrm{dBm})$ compared with DPPM and DMPPM, respectively. Moreover, the effect of changing source wavelength has shown that, at low power, the short wave length based system is better than the long wavelength based ones. For example, at low power, DPPMachieves BER around $4 \times 10^{-3}$ and $8 \times 10^{-3}$ at wavelength $850 \mathrm{~nm}$ and $1550 \mathrm{~nm}$, respectively while at high power, DPPM achieves $6 \times$ $10^{-5}$ and $1 \times 10^{-5}$ at wavelength $850 \mathrm{~nm}$ and $1550 \mathrm{~nm}$ at moderate turbulence.

\section{References}

[1]A. E. Morra, H. S. Khallaf, H. M. H. Shalaby, Z. Kawasaki, Performance analysis of both shot- and thermal-noise limited multipulse PPM receivers in gamma-gamma atmospheric channels, J. Lightw. Technol., vol. 31, no. 19, pp. 3142-3150, Oct. 2013.

[2] M. A. Khalighi, M. Uysal, Survey on free space optical communication: A communication theory perspective, IEEE Communications Surveys Tutorials, vol. 16, pp. 2231-2258, June 2014.

[3]J. Hamkins, B. Moision, Selection of modulation and codes for deepspace optical communications, Proceedings of SPIE, Free-Space Laser Communication Technologies XVI, vol. 5338, pp.123-130, San Jose, CA, Jan. 2004.

[4] A. E. Morra, K. Ahmed, S. Hranilovic, Impact of fiber nonlinear on 5G backhauling via mixed FSO/fiber network, IEEE Access, vol. 5, pp. 19942-19950, Sept. 2017.

[5] M. Najafi, V. Jamali, R. Schober, Optimal relay selection for the parallel hybrid RF/FSO relay channel: Non-buffer-aided and bufferaided designs, IEEE Transactions on Communicions, vol. 65, pp. 2794- 2810, July 2017.

[6] P. V. Trinh, T. C. Thang, A. T. Pham, Mixed mmwave RF/FSO relaying systems over generalized fading channels with pointing errors, IEEE Photonics Journal, vol. 9, no. 1, Feb. 2017.

[7]A. Proke, Modeling of atmospheric turbulence effect on terrestrial FSO link, Radio engineering, vol. 18, no. 1, Apr. 2009.

[8]H. Kawasaki, k. Nosu, MPPM: Amethod for improving the bandutilization efficiency in optical PPM, J. Lightw. Technol., vol. 7, pp. 465-472, Mar. 1989.

[9]S. S. Muhammad, T. Javornik, I. Jelovcan, E. Ghassemlooy, ZabihandLeitgeb, Comparison of hard decision and soft decision channel coded m-ary PPM performance over free space optical links, European Transactions on Telecommunications, vol. 20, no. 8, pp. 746-757, Dec. 2009.

[10]H. Shalaby, Performance of uncoded overlapping PPM under communication constraints, Proceedings of ICC '93 - IEEE International Conference on Communications 1993, Geneva, Switzerland, Switzerland.

[11]D. s. Shiu, J.M. Kahn, Differentialpulse-positionmodulationfor powerefficient optical communication, IEEE Transactionson Communications, vol. 47, no. 8, pp. 1201-1210, Aug. 1999.

[12]T. Wang, Z. Hao, H. Li, W. Zhan, R. Li, Error rate performance of differential multi-pulse position modulation, Cluster Computing,doi: 10.1007/s10586-018-2372-z, pp. 1-9, Mar. 2018 
[13]J. E. Gancarz, H. Elgala, T. D. Little, Overlapping PPM for bandlimited visible light communication and dimming, Journal of Solid State Lighting, vol. 1, May 2015.

[14]F. Xu, M. A. Khalighi, S. Bourennane, Coded PPM and multipulse PPM and iterative detection for free-space optical links, Journal of Optical Communications and Networking, vol. 1, pp. 404-415, Oct. 2009.

[15]Z. Wang, W. D. Zhong, S. Fu, C. Lin, Performance comparison of different modulation formats over free-space optical (FSO) turbulence links with space diversity reception technique, IEEE Photonics Journal, vol. 1, no. 6, pp. 277-285, Dec. 2009.

[16]X. Tang, S. Rajbhandari, W. O. Popoola, Z. Ghassemlooy, E. Leitgeb, S. S. Muhammad, G. Kandus, Performance of BPSK subcarrier intensity modulation free-space optical communications using a lognormal atmospheric turbulence model, 2010 Symposium on Photonics and Optoelectronics, pp. 4-1, 2010.

[17]H. E. Nistazakis, V. Assimakopoulos, G. Tombras, Performance estimation of free space optical links over negative exponential atmospheric turbulence channels, OPTIK-International Journal for Light and Electron Optics, vol. 122, no. 24 , pp. 2191-2194, 2011.

[18]D. A. Guimaraes, Digital transmission: A simulation-aided introduction with vissim/com., Springer, 2009.

[19]ChristenaGhandour, Abdel HalimZekry, Nazmi A. Mohammed, S. Elrabaie, An In-depth Survey of Visible Light Communication Modulating and Coding schemes for Dimming/Flicker Avoidance Technologies, Menoufia J. of Electronic Engineering Research (MJEER), vol. 28, no. 1, Jan. 2019

[20]Z. Ghassemlooy, W. Popoola, S. Rajbhandari, Optical wireless communications : System and channel modelling with matlab, 1st ed. CRC Press, Aug. 2012.

[21] J. Hamkins, B. Moision, Multipulse pulse-position modulation on discrete memoryless channels, IPN Progress Report 42-161, May 2005.

[22]Y. Rahmatallah, S. Mohan, Peak-to-average power ratio reduction in OFDM systems: A survey and taxonomy, IEEE Communications Surveys \& Tutorials, vol. 15, no. 4, pp.1567-1592, Fourth quarter 2013.

[23] F. Xu, M. Khalighi, S. Bourenane, Impact of different noise sources on the performance of PIN- and APD-based FSO receivers, ConTEL 2011-11th Int. Conf. on Telecommun., Graz, Austria, June 2011.

[24]H. Nistazakis, T. Tsiftsis, G. Tombras, Performance analysis of freespace optical communication systems over atmospheric turbulence channels, IET Commun, vol. 3, pp. 1402-1409, Aug. 2009.

[25]R. L. Al-Habash, M. A. Andrews, L. C. Phillips, Mathematical model for irradiance probability density function of a laser beam propagating throughturbulentmedia,OpticalEngineering,vol.40,no.8,pp.5541562, Aug. 2001.

[26]G. Parry, Measurement of atmospheric turbulence induced intensity fluctuations in a laser beam, Opt. Acta: International Journal of Optics, vol. 28, no. 5, pp. 715-728, 1981.

[27]W. F. Site, [online], Available: http:// functions.wolfram.com/. (Jan. 2019).

[28]H. Selmy, H. M. H. Shalaby, Z. Kawasaki, Performance analysis of gradual multi-pulse pulse-position modulation in deep-space optical communications, JOCN, vol. 4, no. 10, pp. 812-820, Oct. 2012. 